\title{
Effects of unweighting on gait kinematics during walking on a lower-body positive- pressure treadmill in patients with hip osteoarthritis
}

Yoshiaki Kataoka ${ }^{1,2}$, Tomohiro Shimizu ${ }^{3^{*}}$ (D, Ryo Takeda ${ }^{4}$, Shigeru Tadano ${ }^{4}$, Yuki Saito ${ }^{1}$, Satoshi Osuka ${ }^{1}$, Tomoya Ishida', Mina Samukawa', Tohru Irie ${ }^{3}$, Daisuke Takahashi ${ }^{3}$, Norimasa Iwasaki ${ }^{3}$ and Harukazu Tohyama ${ }^{1}$

\begin{abstract}
Background: Hip osteoarthritis $(\mathrm{OA})$ is a musculoskeletal condition that makes walking difficult due to pain induced by weight-bearing activities. Treadmills that support the body weight (BW) reduce the load on the lower limbs, and those equipped with a lower-body positive-pressure (LBPP) device, developed as a new method for unweighting, significantly reduce pain in patients with knee OA. However, the effects of unweighting on gait kinematics remain unclear in patients with hip OA. Therefore, we investigated the effects of unweighting on kinematics in patients with hip OA during walking on a treadmill equipped with an LBPP device.

Methods: A total of 15 women with hip OA and 15 age-matched female controls wore a three-dimensional (3-D) motion analysis system and walked at a self-selected speed on the LBPP treadmill. Data regarding self-reported hip pain using a numeric rating scale (NRS) in which the scores 0 and 10 represented no pain and the worst pain, respectively, under three different BW conditions (100, 75, and 50\%) were collected. Moreover, 3-D peak joint angles during gait under each condition were calculated and compared.

Results: In the hip OA group, the NRS pain scores at 50 and 75\% BW conditions significantly decreased compared with that at $100 \%$ BW condition $(50 \%, P=0.002 ; 75 \%, P=0.026)$, and the peak hip extension angle decreased compared with that in the healthy controls $(P=0.044)$. In both groups, unweighting significantly decreased the peak hip $(P<0.001)$ and knee $(P<0.001)$ flexion angles and increased the peak ankle plantar flexion angle $(P<0.001)$ during walking.

Conclusions: Unweighting by the LBPP treadmill decreased pain in the hip OA group but did not drastically alter the gait kinematics compared with that in the control group. Therefore, regarding the use of the LBPP treadmill for patients with hip OA, clinicians should consider the benefits of pain reduction rather than the kinematic changes.
\end{abstract}

Keywords: Osteoarthritis, Walking, Weight-bearing, Gait, Hip joint, Pain

\footnotetext{
* Correspondence: simitom@wg8.so-net.ne.jp

${ }^{3}$ Department of Orthopaedic Surgery, Faculty of Medicine and Graduate

School of Medicine, Hokkaido University, Kita 15, Nishi 7, Kita-ku, Sapporo, Hokkaido 060-8638, Japan

Full list of author information is available at the end of the article
}

(C) The Author(s). 2021 Open Access This article is licensed under a Creative Commons Attribution 4.0 International License, which permits use, sharing, adaptation, distribution and reproduction in any medium or format, as long as you give appropriate credit to the original author(s) and the source, provide a link to the Creative Commons licence, and indicate if changes were made. The images or other third party material in this article are included in the article's Creative Commons licence, unless indicated otherwise in a credit line to the material. If material is not included in the article's Creative Commons licence and your intended use is not permitted by statutory regulation or exceeds the permitted use, you will need to obtain permission directly from the copyright holder. To view a copy of this licence, visit http://creativecommons.org/licenses/by/4.0/ The Creative Commons Public Domain Dedication waiver (http://creativecommons.org/publicdomain/zero/1.0/) applies to the data made available in this article, unless otherwise stated in a credit line to the data. 


\section{Background}

Walking exercises are widely used in individuals with hip osteoarthritis (OA) for rehabilitation $[1,2]$. However, these individuals often have difficulty in walking due to pain and excessive force induced by weight-bearing activities. Treadmills equipped with a lower-body positive-pressure (LBPP) device have been developed to provide precise unweighting during walking $[3,4]$. Because LBPP treadmills reduce the stress induced by ground reaction forces on the lower limbs, unweighting by the LBPP treadmill has shown to significantly reduce pain in patients with $\mathrm{OA}$ and, therefore, has the potential to maintain or enhance aerobic exercise capacity $[5,6]$. In addition, LBPP treadmills reduce the load on the cardiopulmonary function [3], thereby reducing the rate of perceived exertion compared with treadmills with a harness system [7].

Investigating gait kinematics on LBPP for hip OA can provide useful information for clinicians when they apply LBPP exercises. However, as a participant's lower limbs are in a waist-high chamber when using an LBPP treadmill, a conventional motion analysis using an optical method may be difficult, especially for the hip joint, limiting what is known about how unweighting affects gait kinematics. Because of the advances in technology, a wearable-sensor-based three-dimensional (3-D) motion analysis system, which can analyze gait kinematics by seven sensors that consist of triaxial acceleration and gyro sensors, has recently been developed as a tool to analyze gait kinematics [8]. Hence, we thought that we could calculate gait kinematics in participants with hip OA by this system while walking on an LBPP treadmill.

The present study aimed to investigate the use of wearable sensors with an LBPP treadmill and the unweighting effects on 3-D kinematics in participants with hip OA. The present study hypothesized that (1) the participants with OA would report less pain when unweighted by the LBPP treadmill and (2) all participants would exhibit increased peak hip, knee, and ankle joint angles.

\section{Methods}

\section{Participants}

This study was approved by the institutional review board of our university, and informed consent was obtained from all participants. In total, 15 female participants with hip OA and 15 female healthy controls were recruited. The inclusion criteria of the hip OA group were women who were scheduled to undergo unilateral total hip arthroplasty (THA) for treatment of moderate to severe $\mathrm{OA}$ and aged $<85$ years. The severity of OA was determined on radiography according to the Kellgren and Lawrence (KL) grade [9] in the participants with hip OA. The exclusion criteria of the hip OA group included a history of (1) immunosuppression or autoimmune deficiency, (2) inflammatory arthritis, (3) local or systemic infections, (4) knee arthritis and/or total knee arthroplasty, or (5) symptomatic spinal cord disease. The Harris hip score, which includes sections on pain, function, absence or presence of deformity, and passive range of motion and is scored from 0 (worst) to 100 (best), was used to evaluate participants with hip OA. None of the healthy controls had a history of bone fracture or surgery in the lower limbs; history of neurological, respiratory, or cardiovascular diseases; musculoskeletal disorders within the past 6 months; or previous history of trauma.

\section{Gait protocol}

The participants wore specifically designed shorts with sensors while using the LBPP treadmill. The height of the chamber was fixed to accommodate the participant, and sensors from the shorts were attached to the LBPP treadmill. Then, it was set equal to the height of the greater trochanter of the participant's femur (Fig. 1). To determine the correlation of gravity and the internal pressure of the chamber, calibration was performed for each participant as previously described [10]. The participants walked at a self-selected speed on the LBPP treadmill (Anti-Gravity Treadmill M320, AlterG, Inc.,

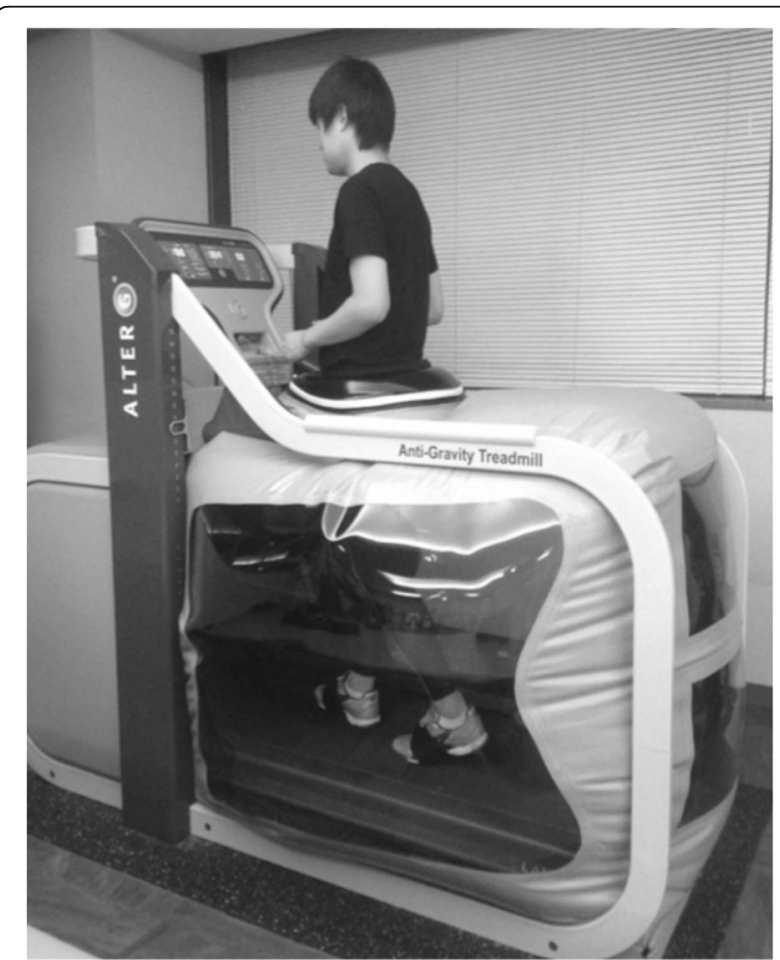

Fig. 1 Lower-body positive pressure (LBPP) treadmill. Participants walk on an LBPP treadmill. The positive pressure inflates the chamber to create traction force on the lower limbs 
Fremont, California, USA) at 100, 75, and 50\% body weight (BW) conditions. The walking speed was consistent across the loading conditions. The participants walked for $30 \mathrm{~s}$ under three conditions selected randomly (100, 75, and 50\% BW) for the testing procedure. Before recording the walking trials, they were asked to familiarize themselves with walking on the LBPP treadmill for 3 min and given $90 \mathrm{~s}$ to adapt to each BW condition. The participants in the hip OA group were asked to assess their hip pain using a numeric rating scale (NRS) in which the scores 0 and 10 represented no pain and the worst pain, respectively [11], during walking under 100,75 , and $50 \% \mathrm{BW}$ conditions. In addition, participants in the control group walked $30 \mathrm{~s}$ again at $100 \%$ BW condition to measure the intra-rater reliability results using minimal detectable changes (MDCs) after the gait protocol.

\section{Data collection using the motion analysis system}

All data collections were performed on the OA side in the OA group and on the dominant leg in the control group. The dominant side in the control group was defined according to which leg the participants used for kicking. Data were collected using a motion analysis system (H-Gait system, Laboratory of Biomechanical Design, Hokkaido University, Sapporo, Japan) where wearable sensors analyzed the 3-D gait kinematics $[8,12]$. Briefly, seven wearable sensor units (TSDN121, ATRPromotions, Inc., Kyoto, Japan), which consisted of triaxial acceleration and gyro sensors, were placed on seven lower-limb body segments (pelvis, right and left thighs, right and left shanks, and right and left feet), as shown in Fig. 2. Acceleration and angular velocity data were collected simultaneously during gait via wireless connection (Bluetooth) in real time. Sensor specifications were the same as those mentioned in the previous studies $[8,12]$.

According to a previous study [12], a calibration test for each participant was performed to measure the acceleration data of the sensors in the upright and inclined positions to calculate the initial inclination of each sensor with respect to the gravity. Before each trial, an initial static phase was acquired in the upright position. When the participants started walking, subsequent 3-D orientations from the initial one were estimated by integrating the angular velocity with the drift removal using the MATLAB software (MathWorks, Natick, MA, USA) [13]. The 3-D angular displacement from the initial upright position was calculated in a quaternion according to a previous study [12]. From these data, the spatiotemporal gait parameters; hip joint angles in the sagittal, coronal, and transverse planes; and knee and ankle joint angles in the sagittal plane during walking under each BW condition were evaluated in each participant. This $\mathrm{H}$-Gait system divided $30 \mathrm{~s}$ of walking into

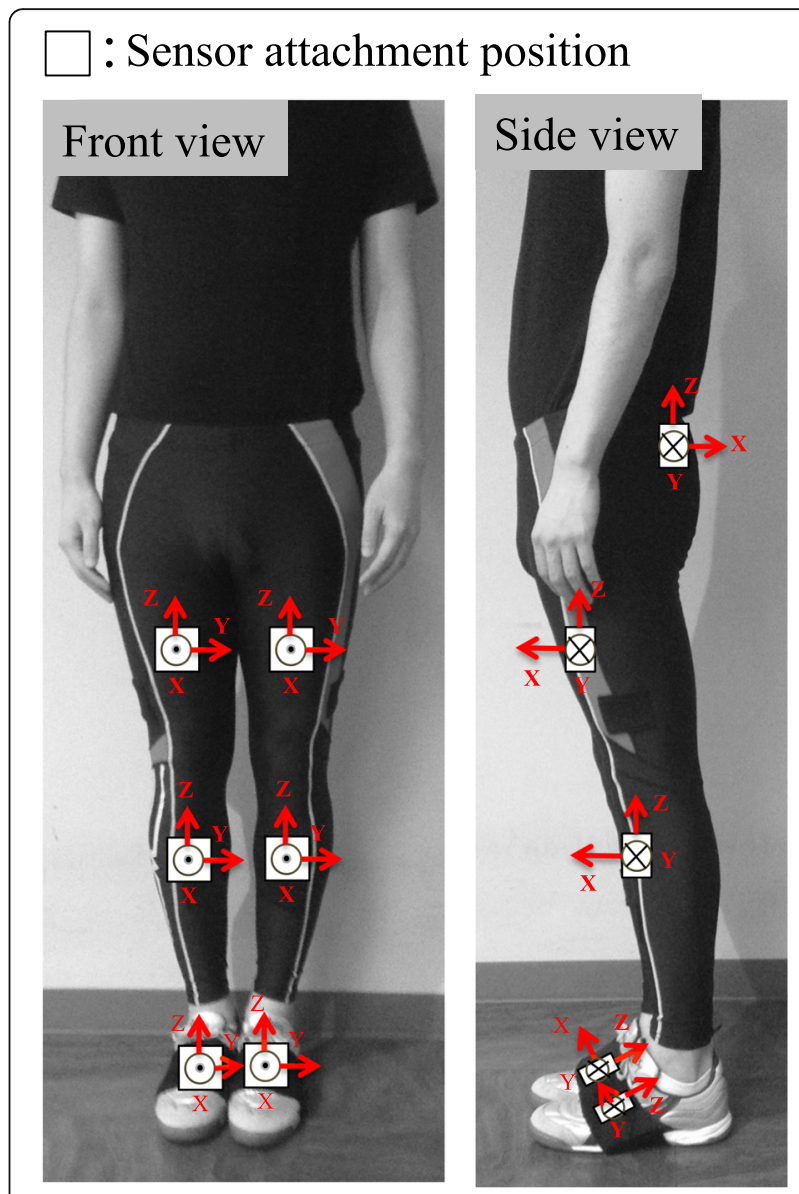

Fig. 2 Sensor attachment position on the lower limbs. Seven wearable sensor units are placed on the pelvis, both thighs, both shanks, and both feet of the participants

gait cycles and calculated the angles of each joint for every gait cycle. The median gait cycle represented by this system during $30 \mathrm{~s}$ of walking under each BW condition was used for analyses. For the gait cycle, one gait cycle from the heel contact to the next heel contact was normalized to $100 \%$. The swing and stance phases were defined using the heel contact and toe off timings of both legs. The heel contact and toe off timings were detected using the peak angular velocity data of the shank as previously reported $[12,14]$. With regard to the validity and reliability of the gait analysis system, Tadano et al. analyzed the kinematics of the lower limbs in walking using the $\mathrm{H}$-Gait system and compared them with that using a camera-based motion analysis system [12]. The correlation coefficients of the hip and knee flexion and ankle dorsiflexion angles were $0.98,0.97$, and 0.78 , respectively.

\section{Statistical analysis}

The demographic characteristics and walking speed between the groups were compared using independent 
Student's t-tests. One-way ANOVAs with post hoc Bonferroni tests were used to investigate the differences in the NRS pain scores during walking under 100, 75, and $50 \%$ BW conditions for the hip OA group. Two-way repeated ANOVAs ( $3 \mathrm{BW}$ conditions $\times 2$ groups) were performed to assess the main effects of the BW conditions (100, 75, and 50\% BW) and groups (control, OA) on the spatiotemporal gait parameters and peak angles of each joint. When the interactions were nonsignificant, the main effects were assessed. If the main effect of the BW condition was statistically significant, post hoc Bonferroni tests were performed to evaluate the significant differences in the spatiotemporal gait parameters and peak angles of each joint among the BW conditions. In addition, the effect sizes for the main effect and interaction between unweighting and group were calculated to determine the magnitude of the differences using eta squared $\left(\eta^{2}\right)$. The significance level was set at 0.05 . Statistical analyses were performed using the IBM SPSS version 17 software (SPSS Inc., Chicago, IL, USA).

\section{Results}

Demographic characteristics, walking speed, and pain

Table 1 summarizes the demographic characteristics of the participants and clinical information in the present study. There were no significant differences in age, height, weight, or walking speed between the hip OA and control groups. The hip OA group included three patients with KL grade 3 and 12 with KL grade 4 .

In the hip OA group, the NRS pain score was significantly lower at $50 \% \mathrm{BW}$ condition than at the $100 \%$ $(P=0.002)$ and $75 \%(P=0.018)$ BW conditions. Moreover, the NRS pain score was significantly lower at $75 \%$ BW condition than at $100 \% \mathrm{BW}$ condition $(P=0.026)$ (Fig. 3).

\section{MDCs on the spatiotemporal gait parameters and the peak hip/knee/ankle joint angles}

MDCs on the spatiotemporal gait parameters and the peak hip/knee/ankle joint angles were as follows: 12.1 $\mathrm{cm}$, for the step length; $18.4 \mathrm{step} / \mathrm{min}$, for the cadence; $5.1^{\circ}$, for the peak hip flexion angle; $3.4^{\circ}$, peak hip

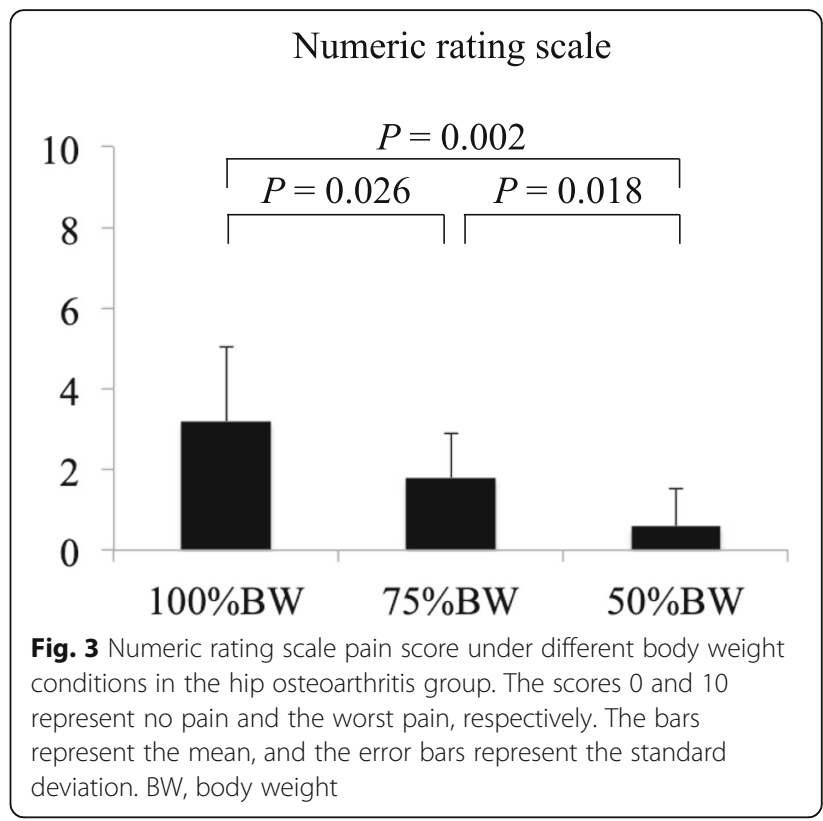

extension angle; $3.9^{\circ}$, peak hip abduction angle; $2.5^{\circ}$, peak hip adduction angle; $3.3^{\circ}$, peak hip external rotation angle; $3.6^{\circ}$, peak hip internal rotation angle; $7.0^{\circ}$, peak knee flexion angle; $3.5^{\circ}$, peak knee extension angle; $5.9^{\circ}$, peak ankle dorsiflexion angle; and $5.2^{\circ}$, peak ankle plantar flexion angle.

\section{Spatiotemporal gait parameters}

For the step length, two-way ANOVA showed a statistical difference between the groups $(P=0.027)$ but not between the BW conditions (100, 75, and 50\%) $(P=0.536)$. No interaction was detected between the groups and BW conditions $(P=0.147)$ (Table 2). Post hoc Bonferroni tests showed that the step length in all BW conditions in the hip OA group decreased compared with that in the control group $(P<0.001)$. For the cadence, two-way ANOVA did not show a significant difference between the groups $(P=0.167)$ and BW conditions $(100,75$, and $50 \%$ $\mathrm{BW})(P=0.219)$. No interaction was detected

Table 1 Demography and walking speed

\begin{tabular}{llrr}
\hline & Hip OA $(\boldsymbol{n}=\mathbf{1 5})$ & Control $(\boldsymbol{n}=\mathbf{1 5})$ & $\boldsymbol{P}$ value \\
\hline Age, years & $60.4(9.6)$ & $61.2(6.3)$ & 0.780 \\
Height, $\mathrm{cm}$ & $152.8(2.9)$ & $155.8(3.7)$ & 0.174 \\
Weight, $\mathrm{kg}$ & $57.1(11.4)$ & $53.5(7.3)$ & 0.329 \\
Walking speed, $\mathrm{km} / \mathrm{h}$ & $1.2(0.3)$ & $1.3(0.4)$ & 0.636 \\
OA KL grade 3 (moderate) & 3 cases & & \\
OA KL grade 4 (severe) & 12 cases & & \\
Harris hip score, point & $45.1(15.3)$ & & \\
\hline
\end{tabular}

Data are presented as mean (standard deviation). OA Osteoarthritis, KL Kellgren and Lawrence 
Table 2 The spatiotemporal gait parameters and peak angles of the hip, knee, and ankle joints

\begin{tabular}{|c|c|c|c|c|c|c|}
\hline Variables & Group & $100 \%$ BW & $75 \%$ BW & $50 \%$ BW & Effect size (group) & Effect size (unweighting) \\
\hline \multirow[t]{2}{*}{ Step length, cm } & Hip OA & $25.3(14.9-35.6)$ & $23.3(13.1-33.6)$ & $24.5(14.5-34.4)$ & 0.83 & $\mathrm{~N} / \mathrm{A}$ \\
\hline & Control & $40.3(20.0-50.7)$ & $46.5(36.2-56.8)$ & $40.7(30.7-50.6)$ & & \\
\hline \multirow[t]{2}{*}{ Hip FLX, degree } & Hip OA & $22.0(16.8-27.1)$ & $20.1(15.2-24.9)$ & $16.2(11.8-20.6)$ & $\mathrm{N} / \mathrm{A}$ & 0.05 \\
\hline & Control & $25.6(20.5-30.8)$ & $24.4(19.6-29.2)$ & $21.0(16.6-25.4)$ & & \\
\hline \multirow[t]{2}{*}{ Hip EXT, degree } & Hip OA & $-0.9(-4.0-2.3)$ & $0.0(-2.9-3.0)$ & $-0.6(-3.4-2.2)$ & 0.04 & N/A \\
\hline & Control & $-4.5(-7.7--1.4)$ & $-3.9(-6.8--0.9)$ & $-4.8(-7.6--2.0)$ & & \\
\hline \multirow[t]{2}{*}{ Hip ADD, degree } & Hip OA & $2.0(0.2-3.8)$ & $1.7(0.1-3.4)$ & $0.1(-1.1-1.4)$ & N/A & 0.01 \\
\hline & Control & $4.6(2.8-6.4)$ & $3.3(1.7-4.9)$ & $2.3(0.9-3.8)$ & & \\
\hline \multirow[t]{2}{*}{ Hip IR, degree } & Hip OA & $7.4(4.5-10.3)$ & $6.3(3.6-9.0)$ & $5.0(2.7-7.2)$ & N/A & 0.01 \\
\hline & Control & $7.4(4.5-10.3)$ & $5.9(3.2-8.5)$ & $6.1(3.8-8.3)$ & & \\
\hline \multirow[t]{2}{*}{ Knee FLX, degree } & Hip OA & $47.2(37.6-56.8)$ & $44.0(34.1-53.8)$ & $39.3(30.8-47.8)$ & $\mathrm{N} / \mathrm{A}$ & 0.11 \\
\hline & Control & $59.9(50.3-69.5)$ & $54.7(44.9-64.6)$ & $51.9(43.4-60.4)$ & & \\
\hline \multirow[t]{2}{*}{ Knee EXT, degree } & Hip OA & $-7.1(-9.8--4.5)$ & $-4.5(-6.5--2.5)$ & $-2.8(-4.8--1.0)$ & N/A & 0.01 \\
\hline & Control & $-3.5(-6.2--0.9)$ & $-3.4(-5.5--1.5)$ & $-2.3(-4.2--0.5)$ & & \\
\hline \multirow[t]{2}{*}{ Ankle PF, degree } & Hip OA & $5.5(-0.1-11.4)$ & $10.0(3.9-16.3)$ & $11.1(4.3-17.9)$ & N/A & 0.08 \\
\hline & Control & $7.0(1.2-12.8)$ & $11.4(5.2-17.6)$ & $14.6(7.8-21.4)$ & & \\
\hline
\end{tabular}

Data are presented as mean $(95 \% \mathrm{Cl})$. OA Osteoarthritis, BW Body weight, FLX Flexion, EXT Extension, ADD Adduction, IR Internal rotation, $P F$ Plantar flexion

between the groups and BW conditions $(P=0.052)$ (Fig. 4).

\section{Effects of unweighting on the peak hip/knee/ankle joint angles}

For the peak hip flexion angle during the swing phase, twoway ANOVA showed a significant difference between the $\mathrm{BW}$ conditions $(P<0.001)$ but not between the groups $(P=$ $0.163)$. No interaction was detected between the groups and
BW conditions $(P=0.910)$ (Fig. 5a). Post hoc Bonferroni tests showed that the peak hip flexion angle at 50\% BW condition in both groups decreased statistically significantly compared with that at $100 \% \mathrm{BW}$ condition (hip OA, $P=$ 0.011; control, $P=0.049$ ). For the peak hip abduction and external rotation angles during the stance phase, two-way ANOVA did not show a significant difference between the groups and BW conditions. No interaction was detected between the groups and BW conditions.

Step length, $\mathrm{cm}$

\section{Cadence, step/min}
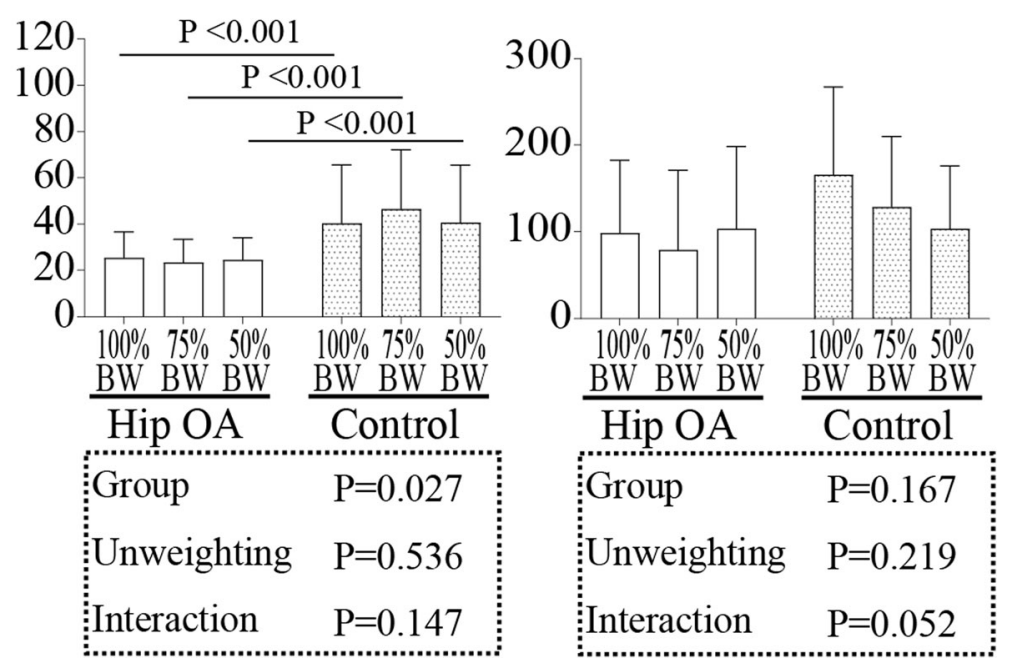

Fig. 4 Spatiotemporal gait parameters under different body weight conditions in the hip osteoarthritis and control groups. The bars represent the mean, and the error bars represent the standard deviation. BW, body weight 


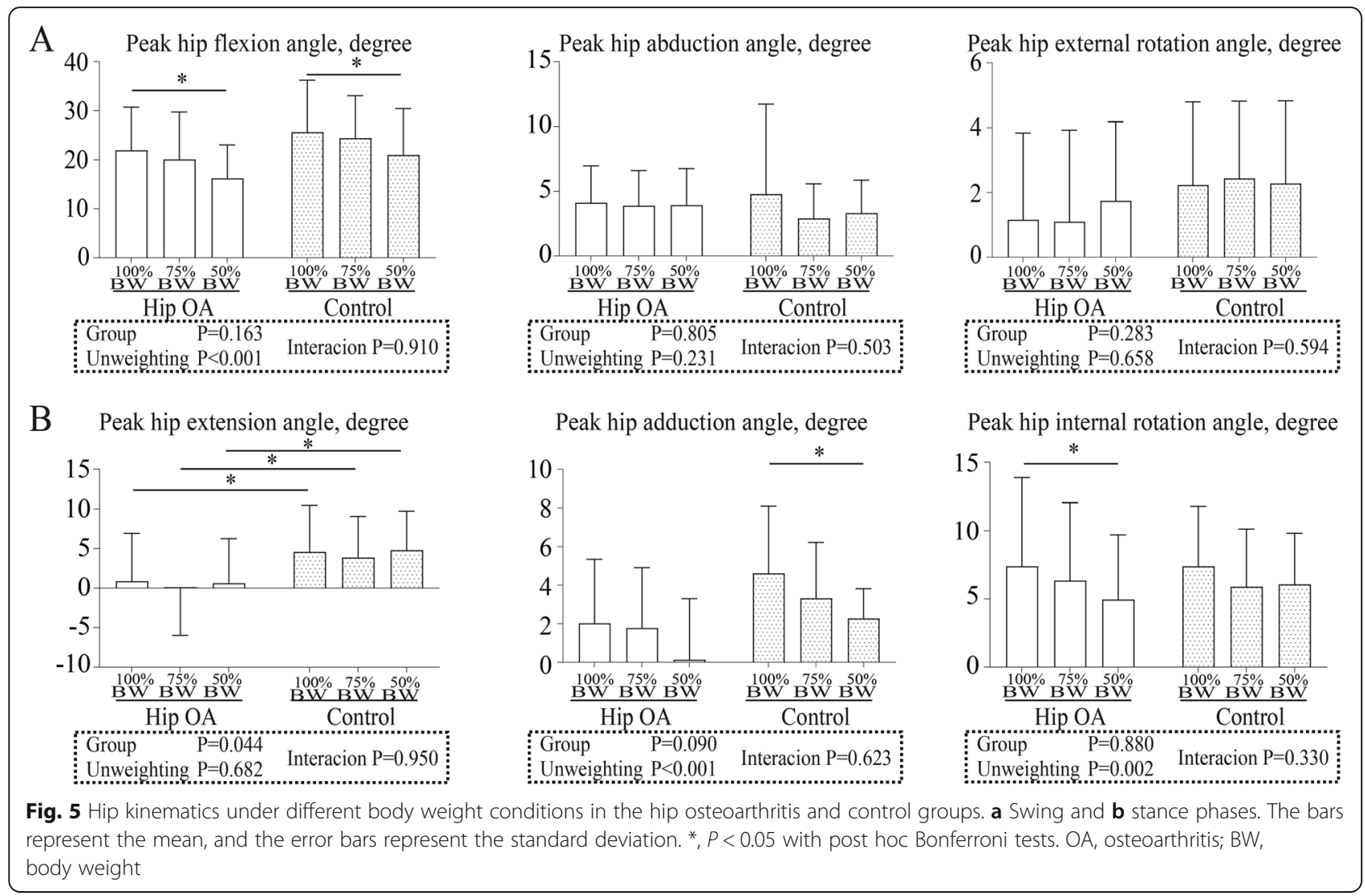

For the peak hip extension angle during the stance phase, two-way ANOVA showed a significant difference between the groups $(P=0.044)$ but not between the BW conditions $(P=0.682)$. No interaction was detected between the groups and BW conditions $(P=0.950)$ (Fig. 5b). Post hoc Bonferroni tests showed that the peak hip extension angle in all BW conditions in the hip OA group decreased compared with that in the control group $(P<0.001)$. For the peak hip adduction and internal rotation angles during the swing phase, two-way ANOVA showed significant differences between the BW conditions (adduction, $P<0.001$; internal rotation, $P=0.002$ ) but not between the groups. No interaction was detected between the groups and BW conditions. Post hoc Bonferroni tests showed that the peak hip adduction angle at $50 \%$ BW condition in the control group decreased statistically significantly compared with that at $100 \% \mathrm{BW}$ condition $(P=0.012)$. Post hoc Bonferroni tests showed that the peak hip internal rotation angle at $50 \% \mathrm{BW}$ condition in the hip OA group decreased statistically significantly compared with that at $100 \% \mathrm{BW}$ condition $(P<0.001)$.

For the peak knee flexion and extension angles, twoway ANOVA showed a significant difference between the BW conditions $(P<0.001)$ but not between the groups. No interaction was detected between the groups and BW conditions (Fig. 6a). Post hoc Bonferroni tests showed that the peak knee flexion angle at $50 \%$ BW condition in both groups decreased statistically significantly compared with that at $100 \% \mathrm{BW}$ condition (hip OA, $P=0.002$; control, $P=0.002$ ) and the peak knee extension angle at 75 and $50 \%$ BW conditions in the hip OA group decreased statistically significantly compared with that at $100 \% \mathrm{BW}$ condition $(75 \%$ vs. $100 \% \mathrm{BW}, P=$ $0.029 ; 50 \%$ vs. $100 \%$ BW, $P<0.001)$.

For the peak ankle plantar flexion angle, two-way ANOVA showed a significant difference between the BW conditions $(P<0.001)$ but not between the groups. No interaction was detected between the groups and BW conditions (Fig. 6b). Post hoc Bonferroni tests showed that the peak ankle plantar flexion angle at $50 \%$ BW condition in both groups increased statistically significantly compared with that at $100 \% \mathrm{BW}$ condition (hip OA, $P=0.020$; control, $P=0.001$ ).

Table 2 summarizes the effect sizes of each parameter that two-way ANOVA showed significant differences in the present study. The effect size of the step length between the groups was large $\left(\eta^{2}=0.83\right)$. The effect sizes of the peak knee flexion and ankle plantar flexion angles were medium $\left(\eta^{2}=0.11\right.$ and $\left.\eta^{2}=0.08\right)$. The effect sizes of other parameters were small. 
A

Peak knee flexion angle, degree

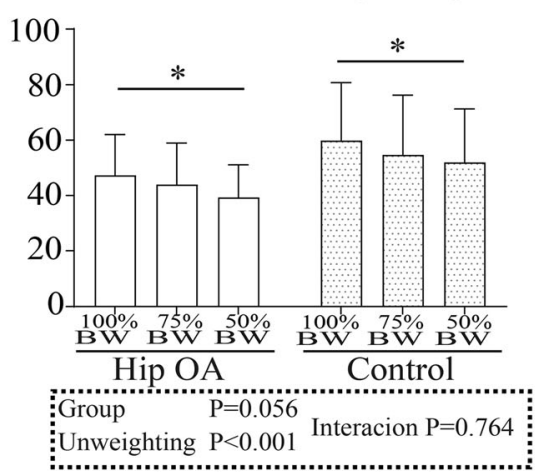

B

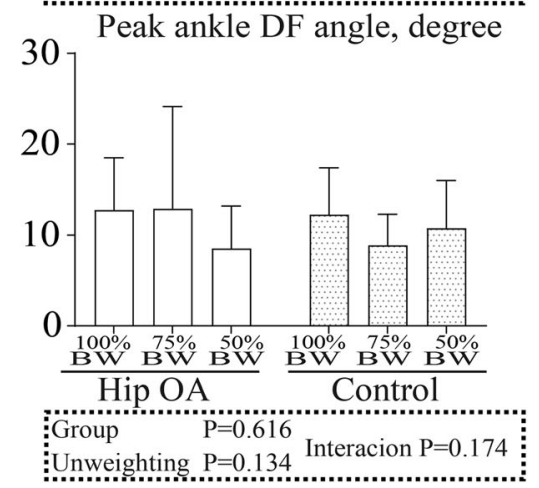

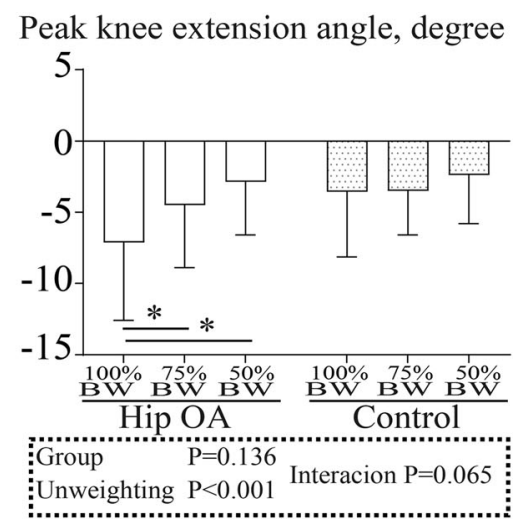

Peak ankle PF angle, degree

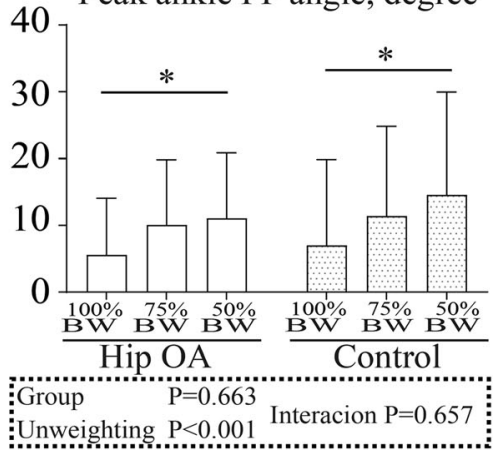

Fig. $\mathbf{6}$ Kinematics of the $\mathbf{a}$ knee and $\mathbf{b}$ ankle joints under different body weight. The bars represent the mean, and the error bars represent the standard deviation. * $P<0.05$ with post hoc Bonferroni tests. OA, osteoarthritis; BW, body weight; DF, dorsiflexion; PF, plantar flexion

\section{Discussion}

In the present study, we first investigated the NRS pain score and gait kinematics during walking on the LBPP treadmill in the participants with hip OA. As expected, this study showed that unweighting by the LBPP treadmill decreased the NRS pain score among the patients with hip OA. Although the LBPP altered the gait kinematics, there were no significant differences between the hip OA and control groups, suggesting that the LBPP treadmill is desirable for decreasing pain after an aerobic exercise rather than the alteration of gait kinematics among patients with hip OA. This finding is consistent with those of a previous study that used the LBPP treadmill to assess acute knee pain during weight-bearing exercise in a population of overweight patients with knee OA [5]. Because gait impairments due to hip pain lead to decreased endurance and muscle strength in the lower limbs, it is clinically important for these patients to perform gait training under safe and comfortable conditions $[3,7]$ with less load on the hip joint. To reduce gait alterations during the LBPP training, the $75 \% \mathrm{BW}$ condition may be useful for participants with hip OA, as this condition significantly reduced pain but did not significantly affect the gait kinematics.

The findings of the present study revealed that contrary to our expectation, unweighting significantly decreased the peak hip and knee flexion angles and increased the peak ankle plantar flexion angle during walking on the LBPP treadmill in both hip OA and control groups. The finding that the peak hip and knee flexion angles decreased during the swing phase under the unweighting condition is consistent with that of previous reports using a treadmill with a harness system $[15,16]$. These kinematic changes during gait could be explained by the higher center of gravity due to traction force. Therefore, this study suggests that unloading treadmill walking does not drastically alter the gait kinematics among patients with hip OA and clinicians should consider these unweighting effects on gait kinematics with regard to the use of the LBPP treadmill for patients with hip OA.

The significant difference in the step length between the hip OA and control groups in the present study was large effect size $\left(\eta^{2}=0.83\right)$ and may reflect the characteristics of patients with hip OA during walking. More specifically, the shorter step length in the hip OA group than in the control group observed in this study was consistent with that in previous studies that showed that the participants with hip OA walked with 7-10\% shorter step length than the age-matched control group $[17,18]$. Although the finding of a lower peak extension angle during the stance phase in the hip OA group compared 
with that in the control group is also consistent with those of previous studies $[19,20]$, the effect size between groups was small $\left(\eta^{2}=0.04\right)$, suggesting that this difference between groups is within the measurement error. Therefore, unweighting by the LBPP treadmill alter the gait kinematics for all participants, not just participants with hip OA. The $\mathrm{H}$-Gait system employs gravity to determine the segment position and orientation. Considering that the LBPP treadmill uses a pressurized chamber to decrease the BW and does not alter gravity, we believe that this $\mathrm{H}$-Gait system could accurately address the kinematics during walking on the LBPP treadmill. However, because this H-Gait system has not yet been validated for use in an altered gravity environment or with the LBPP treadmill, the future study should address sensor adjustment to account for an altered gravity environment.

This study has several limitations. First, only the effects of unweighting on the kinematics of the lower limbs were investigated, rather than including the effects of unweighting on the kinematics of the trunk and upper limbs. Second, this system may have a larger measurement error compared with a camera-based system such as a Vicon system. However, we believed that wearable sensors are an excellent application for this investigation as the treadmill design makes it difficult to acquire motion analysis data using traditional skin marker motion analysis technologies. Third, the present study targeted participants with the moderate or severe hip OA that will be received THA within 3 months of data collection; therefore, unweighting during gait could affect hip pain differently for participants with early stage hip OA. Future study should investigate the training effect of LBPP among patients with early stage hip OA.

\section{Conclusions}

Unweighting by the LBPP treadmill decreased pain in the hip OA group but did not drastically alter the gait kinematics compared with that in the control. Therefore, regarding the use of the LBPP treadmill for patients with hip OA, clinicians should consider the benefits of pain reduction rather than the kinematic changes.

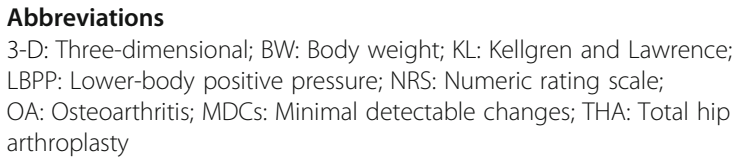

\section{Acknowledgments}

We thank Dr. Tsuyoshi Asano for recruiting the patients.

\section{Authors' contributions}

YK, YS, and SO collected the data. YK, TS, TI, MS, and HT designed the study and drafted the manuscript. YK, RT, and ST performed data processing. YK,
TS, TI, DT, NI, and HT participated in designing the study. All authors read and approved the final manuscript.

\section{Funding}

This work was financially supported, in part, by Harada Electronics Industry Ltd. and Nipro Corporation. The funding bodies played no role in the design of the study and collection, analysis, and interpretation of data and in writing the manuscript.

\section{Availability of data and materials}

The datasets used and/or analyzed during the current study available from the corresponding author on reasonable request.

\section{Ethics approval and consent to participate}

This study was approved by the Hokkaido University Institutional Review Board (\#015-0096), and written informed consent for participation in the study was obtained from all participants.

\section{Consent for publication}

All subjects participating in the image acquisition signed the consent form.

\section{Competing interests}

The authors declare that they have no competing interests.

\section{Author details}

${ }^{1}$ Faculty of Health Sciences, Hokkaido University, Kita 12, Nishi 5, Kita-ku, Sapporo 060-0812, Japan. ${ }^{2}$ Department of Rehabilitation, Health Sciences University of Hokkaido Hospital, 2-5 Ainosato, Kita-ku, Sapporo 002-8072, Japan. ${ }^{3}$ Department of Orthopaedic Surgery, Faculty of Medicine and Graduate School of Medicine, Hokkaido University, Kita 15, Nishi 7, Kita-ku, Sapporo, Hokkaido 060-8638, Japan. ${ }^{4}$ Faculty of Engineering, Hokkaido University, Kita 12, Nishi 8, Kita-ku, Sapporo 060-8628, Japan.

Received: 2 April 2020 Accepted: 23 December 2020

Published online: 08 January 2021

\section{References}

1. Bieler T, Siersma V, Magnusson SP, Kjaer M, Christensen HE, Beyer N. In hip osteoarthritis, Nordic walking is superior to strength training and homebased exercise for improving function. Scand J Med Sci Sports. 2017;27(8): 873-86.

2. Solomonow-Avnon D, Herman A, Levin D, Rozen N, Peled E, Wolf A. Positive outcomes following gait therapy intervention for hip osteoarthritis: a longitudinal study. J Orthop Res. 2017:35(10):2222-32.

3. Cutuk A, Groppo ER, Quigley EJ, White KW, Pedowitz RA, Hargens AR. Ambulation in simulated fractional gravity using lower body positive pressure: cardiovascular safety and gait analyses. J Appl Physiol (1985). 2006; 101(3):771-7.

4. Patil S, Steklov N, Bugbee WD, Goldberg T, Colwell CW Jr, D'Lima DD. Antigravity treadmills are effective in reducing knee forces. J Orthop Res. 2013; 31(5):672-9.

5. Takacs J, Anderson JE, Leiter JR, MacDonald PB, Peeler JD. Lower body positive pressure: an emerging technology in the battle against knee osteoarthritis? Clin Interv Aging. 2013;8:983-91.

6. Peeler J, Christian M, Cooper J, Leiter J, MacDonald P. Managing knee osteoarthritis: the effects of body weight supported physical activity on joint pain, function, and thigh muscle strength. Clin J Sport Med. 2015;25(6): $518-23$

7. Ruckstuhl H, Kho J, Weed M, Wilkinson MW, Hargens AR. Comparing two devices of suspended treadmill walking by varying body unloading and Froude number. Gait Posture. 2009;30(4):446-51.

8. Tadano S, Takeda R, Sasaki K, Fujisawa T, Tohyama H. Gait characterization for osteoarthritis patients using wearable gait sensors (H-gait systems). J Biomech. 2016;49(5):684-90.

9. Kellgren JH, Lawrence JS. Radiological assessment of osteo-arthrosis. Ann Rheum Dis. 1957:16(4):494-502

10. Sainton P, Nicol C, Cabri J, Barthelemy-Montfort J, Chavet P. Kinetics and muscle activity patterns during unweighting and reloading transition phases in running. PLoS One. 2016;11(12):e0168545.

11. Hjermstad MJ, Fayers PM, Haugen DF, Caraceni A, Hanks GW, Loge JH, Fainsinger R, Aass N, Kaasa S, European Palliative Care Research C. studies 
comparing numerical rating scales, verbal rating scales, and visual analogue scales for assessment of pain intensity in adults: a systematic literature review. J Pain Symptom Manag. 2011;41(6):1073-93.

12. Tadano S, Takeda R, Miyagawa H. Three-dimensional gait analysis using wearable acceleration and gyro sensors based on quaternion calculations. Sensors (Basel). 2013;13(7):9321-43.

13. Takeda R, Lisco G, Fujisawa T, Gastaldi L, Tohyama H, Tadano S. Drift removal for improving the accuracy of gait parameters using wearable sensor systems. Sensors (Basel). 2014;14(12):23230-47.

14. Aminian K, Najafi B, Bula C, Leyvraz PF, Robert P. Spatio-temporal parameters of gait measured by an ambulatory system using miniature gyroscopes. J Biomech. 2002;35(5):689-99.

15. Finch L, Barbeau H, Arsenault B. Influence of body weight support on normal human gait: development of a gait retraining strategy. Phys Ther. 1991:71(11):842-55 discussion 855-846.

16. Threlkeld AJ, Cooper LD, Monger BP, Craven AN, Haupt HG. Temporospatial and kinematic gait alterations during treadmill walking with body weight suspension. Gait Posture. 2003;17(3):235-45.

17. Watelain E, Dujardin F, Babier F, Dubois D, Allard P. Pelvic and lower limb compensatory actions of subjects in an early stage of hip osteoarthritis. Arch Phys Med Rehabil. 2001;82(12):1705-11.

18. Constantinou M, Loureiro A, Carty C, Mills P, Barrett R. Hip joint mechanics during walking in individuals with mild-to-moderate hip osteoarthritis. Gait Posture. 2017;53:162-7.

19. Eitzen I, Fernandes L, Nordsletten L, Risberg MA. Sagittal plane gait characteristics in hip osteoarthritis patients with mild to moderate symptoms compared to healthy controls: a cross-sectional study. BMC Musculoskelet Disord. 2012;13:258.

20. Rutherford D, Moreside J, Wong I. Knee joint motion and muscle activation patterns are altered during gait in individuals with moderate hip osteoarthritis compared to asymptomatic cohort. Clin Biomech (Bristol, Avon). 2015;30(6):578-84

\section{Publisher's Note}

Springer Nature remains neutral with regard to jurisdictional claims in published maps and institutional affiliations.

Ready to submit your research? Choose BMC and benefit from:

- fast, convenient online submission

- thorough peer review by experienced researchers in your field

- rapid publication on acceptance

- support for research data, including large and complex data types

- gold Open Access which fosters wider collaboration and increased citations

- maximum visibility for your research: over $100 \mathrm{M}$ website views per year

At $\mathrm{BMC}$, research is always in progress.

Learn more biomedcentral.com/submissions 